\title{
Ejemplificación de metodología mixta para el análisis del uso de entornos blended learning en docentes universitarios
}

\author{
Antonio Víctor Martín-García, María Cruz Sánchez-Gómez, Bárbara Gutiérrez Pérez \\ avmg@usal.es, mcsago@usal.es, barbaragutierrez@usal.es \\ Universidad de Salamanca, 37008. Facultad de Educación. Salamanca, España
}

DOI: 10.17013/risti.33. 16-31

\begin{abstract}
Resumen: El objetivo de este trabajo es averiguar a partir de las experiencias y percepciones de profesores universitarios, las principales expectativas, actitudes, demandas, ventajas y desventajas de la aceptación -y adopción- de las metodologías de Blended Learning (BL) en la enseñanza superior. El trabajo empírico se realizó siguiendo un diseño metodológico mixto de tipo derivativo secuencial de equivalencia de status. La información cuantitativa fue recogida con un cuestionario, elaborado ad hoc, al que contestaron 980 profesores universitarios; de éstos, 86 manifestaron de forma abierta su opinión sobre estas metodologías. Para integrar los resultados de los análisis CUANT-CUAL se realizó un análisis DAFO en el que se ponen de manifiesto, a juicio de los docentes participantes en el estudio, las debilidades, amenazas, fortalezas y oportunidades de la enseñanza B-learning, a nivel interno y externo de las instituciones universitarias
\end{abstract}

Palabras-clave: Blended learning, Educación superior, Mixed-methods, modelo TAM, DAFO

\section{Exemplification of Mixed Methodology for the Analysis of the Use of Blended Learning Environments amongst University Teachers}

\begin{abstract}
The objective of this work is to find out, through the experiences and perceptions of university teachers, what the main expectations, attitudes, demands, advantages and disadvantages of the acceptance -and adoption- of Blended Learning (BL) methodologies are in higher education. The empirical work was carried out following a mixed methodological design of sequential derivative type of status equivalence. The quantitative information was collected with an ad hoc questionnaire to which 980 university teachers replied; of these, 86 openly expressed their opinion about these methodologies. In order to integrate the results of the QUANT-QUAL analysis, a SWOT analysis was carried out in which university teachers expressed the weaknesses, threats, strengths and opportunities of Blended Learning at universities - at both an internal and external level.
\end{abstract}

Keywords: Blended learning, Higher education, Mixed-methods, TAM model, SWOT 


\section{Introducción}

El fuerte desarrollo de tecnologías digitales aplicadas a la educación está favoreciendo un proceso cada vez más extendido de integración y convergencia entre la enseñanza tradicional cara a cara y la formación on line, bajo la expresión comúnmente aceptada de blended learning (BL). Tanto desde una perspectiva pedagógica y de tecnología educativa, como desde los modelos de negocio y gestión de las universidades, existe un creciente interés en conocer la forma de difundir y extender estos entornos mixtos de aprendizaje (Cavanaugh, Hargis, y Mayberry, 2016; Dziuban, Graham, Moskal, Norberg, \& Sicilia, 2018; Ibáñez, de Benito, Garcies, \& Cervera, 2018 etc.). El proceso de aceptación y difusión de este tipo de sistemas formativos pasa por valorar las ventajas objetivas que presenta tanto sobre la modalidad cara a cara como sobre la enseñanza a distancia (Ginns, \& Ellis, 2009; Lai, Lam y Lim, 2016, Lim, \& Morris, 2009; etc ), precisa también desarrollar diseños específicos de BL en función de las características y diversidad de los estudiantes (Boelens, Voet, \& De Wever, 2018), pero sobre todo requiere conocer cuáles son las expectativas, valoraciones y actitudes de profesores y estudiantes sobre la formación basada en entornos combinados, puesto que se entiende que en la base de esa actitud y creencias del sujeto hacia el uso de esta innovación tecno-educativa está la clave del éxito de su implementación y aceptación efectiva (Bliuc, Casey, Bachfischer, Goodyear, \& Ellis,2012; Graham, Woodfield, \& Harrison, 2013; Martín-García, SánchezGómez, Costa, 2019).

En esta línea, nuestro estudio se sitúa conceptualmente en los denominados modelos de expectativa-valor, para los que la actitud general y las creencias principales sobre un objeto dado mediatizan y condicionan su intención de uso, así como también la propia acción o uso real del mismo. Es decir, en el caso de BL, se hipotetiza que un profesor valorará las ventajas o mejoras para hacer más eficiente su trabajo que puede suponer implementar un sistema BL, lo que determinará su uso o no uso. El marco teórico más comúnmente utilizado en este tipo de estudios son los Modelos de Aceptación Tecnológica (Technological Aceptation Model, TAM) o variantes del mismo como the Unified Theory of Acceptance and Use of Technology (UTAUT), inicialmente construidos para predecir el uso de nuevos elementos tecnológicos en ambientes organizacionales y de e-goverment (Barrera-Barrera, Rey-Moreno \& Medina-Molina, 2018; Ngai, Poon, \& Chan. 2007), pero también con una aplicación muy extendida en estudios de mercado y consumo y, en menor medida, para el análisis particular de la aceptación de determinadas innovaciones educativas (Imtiaz, \& Maarop, 2014), en general relacionadas con la formación on line (Aliyu, Arasanmi, \& Ekundayo, 2019; Lee, Yoon, \& Lee, 2009; Pynoo, Tondeur, Van Braak, Duyck, Sijnave, \& Duyck, 2012; Selim, 2007; Teo, 2011)

La ventaja de utilizar estos modelos está en que ofrecen un marco comprensivo de dependencia entre las variables consideradas y de éstas respecto a las variables dependientes principales, que son la intención de uso (BI_bl) y la conducta real de Uso (B_bl) ofreciendo así el soporte conceptual de referencia ampliamente presente en la literatura especializada en este campo (Imtiaz, \& Maarop, 2014; Lee, Kozar, \& Larsen, 2003; Maruping \& Bala, 2008; Marangunić, \& Granić, 2015; Venkatesh, Morris, Davis, \& Davis, 2003; Venkatesh \& Bala, 2008; Scherer, Siddiq, \& Tondeur, 2019). 


\subsection{Los Modelos de Aceptación Tecnológica}

Los Modelos de Aceptación Tecnológica (TAM) se derivan de la TRA (Theory of Reasoned Action) propuesta por Fishbein y Ajzen en los años 70 y de la Teoría del Comportamiento Planeado (Theory of Planned Behavior, TPB), variante que introduce I. Ajzen en 1991. Ambas defienden la idea de que gran parte de la conducta humana tiene una base racional (son modelos de expectativa-valor) determinada por la intención de un sujeto de realizar dicha acción o conducta. La intención conductual (IC) (definida como la probabilidad subjetiva de realizar esa conducta) está determinada a su vez por la Actitud hacia la conducta (AT), configurada en base a una serie de creencias principales sobre las consecuencias de realizar esa acción y, por otro, la Norma Subjetiva (NS) que es la percepción por parte del sujeto de que personas importantes para él aprobarán o no que realice dicha conducta. El modelo TAM planteado originalmente por Davis en los años 80, (Davis, Bagozzi, \& Warshaw, 1989) para el estudio de conductas de uso de diferentes tipos de tecnologías, lo que hace es agrupar las creencias que dan lugar a la Actitud en dos tipos: la Utilidad Percibida (UP, Perceived Usefulness) y la Facilidad percibida de uso (Perceived Ease of Use, PEOU). La sucesiva replicación empírica del modelo TAM, particularmente en ambientes corporativos y de organizaciones laborales, hizo ver que el efecto de UP y PEOU era directo sobre IC, sin necesidad de pasar por la Actitud. Se entiende así que independientemente de que un trabajador tenga una actitud positiva o negativa sobre el uso de determinado sistema, usará éste si valora fuertemente las ventajas y beneficios que le reportará su uso (UP), sumado a la idea que el uso de dicho dispositivo o sistema sea valorado por éste como relativamente fácil de usar (PEOU). Por otro lado, el efecto de la opinión de otros sobre la conducta del sujeto perdía valor en los estudios empíricos y esta variable acabó también por desaparecer de las nuevas versiones de los modelos TAM. Por lo tanto, dos únicas variables (UP y PEOU) podían explicar, en los modelos TAM iniciales, en torno al 40, 50 \% de la variabilidad de la conducta (B).

Las sucesivas extensiones del modelo, denominadas TAM2 (Venkatesh \& Davis ,200o) y TAM3 (Venkatesh \& Bala, 2008) responden a la necesidad de mejorar el poder predictivo del modelo inicial, en especial cuando se amplía su uso a diferentes tipos de contextos y conductas de uso, como pueden ser sistemas complejos como los educativos, en los que el componente tecnológico es importante, pero evidentemente no el único. Por ello, el modelo ha ido dando cabida a nuevas variables explicativas relacionadas con factores afectivos, características individuales de los usuarios o factores relacionados con el efecto o influencia social y del contexto. En el caso concreto de los entornos de formación, algunos de estos modelos TAM han explorado nuevas variables, concretamente: apoyo organizacional, autoeficacia relacionada con el uso de TIC, experiencia previa, la ambigüedad de la tarea (task equivocality), satisfacción personal y características individuales (Al-Busaidi, 2013), o también factores como por ejemplo el estilo de aprendizaje de los alumnos (Chen, 2011), la percepción de la autoeficacia en el uso de sistemas con fuerte componente tecnológico, la percepción del control externo, la ansiedad ante contextos tecnologizados en el aula (Konak, Kulturel-Konak, Nasereddin, \& Bartolacci, 2017)

Todo ello ha generado otra serie de propuestas que modelizan el posible efecto de estos nuevos factores explicativos sobre IC (BI, Behavioral Intention). Aparecen así diferentes 
tipos de explicaciones y modelos, con una base teórica similar, pero con factores explicativos diferentes, por lo que resulta necesario adaptar el modelo TAM general a las circunstancias particulares de estudio en cada caso.

\subsection{El uso de modelos mixtos sobre la percepción de Blended learning}

La gran mayoría de estudios realizados sobre adopción de innovaciones tecnológicas tienen su apoyo metodológico en metodología cuantitativa, en su mayor parte utilizando análisis basados en ecuaciones estructurales (SEM) y con fuerte aparato estadísticos. El uso del método mixto para estudios en los que se utilizan como base los modelos TAM no es especialmente abundante en la literatura. Algunos trabajos, como por ejemplo el realizado por Fei Wu (2011) llaman la atención sobre las limitaciones de diseños basados exclusivamente en el análisis cuantitativo, encontrando aspectos muy positivos en el enfoque cualitativo para refinar la comprensión de algunas variables del modelo TAM, en particular el sentido dado por los sujetos a la Utilidad Percibida. De igual modo, otros trabajos apoyan también el uso de metodología mixta (Kim, Kankanhalli, \& Lee, 2016). Por su parte, trabajos recientes apuntalan una línea de análisis para el estudio de la adopción de la modalidad blended learning desde la perspectiva de los métodos mixtos (Ilic, Hart, Fiddes, Misso, \& Villanueva; Al-Azawei, Parslow, \& Lundqvist, K., 2017; Poon, 2013; Ilic, Nordin, Glasziou, Tilson, \& Villanueva, 2013).

En nuestro trabajo, planteamos un diseño que combina ambas metodologías, tratando de evaluar y medir las principales variables del modelo TAM y valorar la percepción de los docentes sobre la utilidad, en términos de ventajas y mejoras, que tiene utilizar un sistema formativo basado en BL, el modo cómo perciben los profesores si se trata de un sistema relativamente fácil de implementar, motivador para los estudiantes o, por el contrario, dada su complejidad, resulta ininteligible y genera incomodidad o malestar para el desempeño docente. De igual modo, se valoran aspectos como en qué medida se percibe la influencia o presión social ejercida por parte de las autoridades académicas, los colegas o los propios estudiantes para acelerar el uso de la innovación, o en qué medida los profesores perciben la existencia de condiciones y recursos materiales y técnicos que facilitan o dificultan su uso. A partir de aquí, la pregunta de investigación se concreta en tratar de determinar cuáles de estos factores principales están asociados a la implementación de uso de esta modalidad formativa (BL), en todo caso, qué factores pueden predecir la intención de uso de la misma en el contexto de las universidades públicas españolas.

\section{Método}

\subsection{Estudio cuantitativo: participantes, medida de variables y análisis}

En el estudio han participado un total de 980 profesores de universidades públicas de todo el territorio español quienes respondieron a un cuestionario enviado a través de internet. De ellos, 481 profesores y 499 profesoras; el 36,5 \% menores de 44 años, el 38,1 entre 45 y 54 y el 25, 4 \% mayores de 55 años; el 65\% son profesores ordinarios $(n=637)$. El 71,8 \% tiene más de 10 años de experiencia docente en la universidad. Por Rama de Conocimiento, la muestra se dividió en Ciencias Sociales y Jurídicas (390 docentes); 
Ciencias de la Salud ( $=154)$, Arquitectura e Ingeniería $(n=132)$, Ciencias $(n=150)$ y Arte y Humanidades $(n=156)$. Por último, el 41,0\% de la muestra afirma haber recibido cursos de formación en B-Learning, mientras que el 48,7\% indica explícitamente no haber recibido formación específica en esta materia.

El cuestionario aplicado se construyó en base a valoración de creencias de los profesores sobre la conveniencia, efectos, posibles resultados, dificultades, etc. de utilizar la metodología BL en docencia universitaria. Para la construcción de las diferentes escalas de medida se tomó como marco teórico los modelos de Aceptación Tecnológica (TAM) para la definición de las variables principales: Utilidad Percibida (PU); Facilidad percibida de Uso (PEOU), Actitud hacia el uso de BL (AT_bl); Condiciones Facilitadoras (CF) e Intención de uso de BL ( IP_bl) , utilizando ítems adaptados de la literatura especializada sobre el tema (Ajzen, 2002; Brown, Dennis, \& Venkatesh, 2010; Davis et al., 1989; Pynoo, Devolder, Tondeur, Van Braak, Duyck, \& Duyck, 2011; Šumak, \& Šorgo, 2011; Teo, Fan \& Du, , 2015; Venkatesh et al., 2008; Venkatesh, et al., 2016).

Mediante la técnica de Análisis Factorial Confirmatorio se evaluó la idoneidad de los indicadores en las variables latentes, es decir, la fiabilidad de los ítems imponiendo una restricción sobre cada una de las cargas estandarizadas a un valor de o,70 o más. Para analizar la validez convergente se ha tenido en cuenta la varianza media extraída (AVE superior a $0,5, \mathrm{AVE}$ : $\mathrm{UP}=.71, \mathrm{PEOU}=.57 ; \mathrm{AT}=, 59 ; \mathrm{CF}=.55 ; \mathrm{BI}=, 60 ;$ ) y cargas factoriales con valores superiores a 0,7 para la fiabilidad compuesta, FCC: $\mathrm{UP}=, 90 ; \mathrm{PEOU}=0,84$; $\mathrm{AT}=, 81 ; \mathrm{CF}=.7 \mathrm{O} ; \mathrm{BI}=, 88)$. Finalmente, para analizar la validez discriminante, se verificó que la raíz cuadrada de la varianza media extraída para cada constructo (AVE) era superior a las correlaciones entre ésta y las otras variables del modelo. Los indicadores de ajuste del modelo son los siguientes: $\chi 2$ /d.f $(\mathrm{CMIN} / \mathrm{DF})=3,26 ; \mathrm{CFI}=0,953 ; \mathrm{TLI}=0,943$; $\mathrm{IFI}=0,954 ; \mathrm{PCFI}=0,777 ; \mathrm{NFI}=0,934 ; \mathrm{RMSEA}=0,048$. Además, se realizaron contrastes estadísticos entre las variables TAM y las variables de clasificación consideradas en el estudio. Ofrecemos a continuación el contraste por ramas de conocimiento para detectar las posibles diferencias entre las titulaciones de Arquitectura_Ingeniería; Arte y humanidades; Ciencias Sociales y Jurídicas; Ciencias y Ciencias de la Salud. Posteriormente buscaremos los significados de estas diferencias en los comentarios que los docentes universitarios hicieron en las respuestas abiertas.

\subsection{Estudio cualitativo: Participantes, técnicas de recogida de información y análisis}

Como se ha explicado anteriormente el estudio cuantitativo se enmarca dentro de un diseño ex--post-facto, de tipo descriptivo y comparativo que busca descubrir relaciones causa-efecto entre variables, complementado con análisis cualitativo de preguntas abiertas. Al tratarse de un estudio de ámbito nacional, se optó por un sistema de encuesta autoadministrada a través de internet para facilitar un mejor acceso a la población objeto de estudio y una mayor rapidez en la recogida de datos. A las preguntas abiertas respondieron 86 docentes universitarios. De esta forma se pudo describir el uso del B_learning, los cambios producidos en los procesos de enseñanza aprendizaje y la valoración personal que los docentes hacían de los mismos. Se optó por establecer varios grupos de profesores en función del ámbito disciplinar en el que se sitúan (Ciencias sociales y Jurídicas, Arte y Humanidades, Ciencias Experimentales y Ciencias de la 
Salud utilizando esta variable para el contraste y ejemplificación del proceso de análisis mixto CUANTI-CUALI. Tras la lectura de las respuestas abiertas, se realizó un análisis de contenido clásico que consta de una primera fase de identificación de dimensiones relevantes o categorización que se visualizan en el mapa conceptual siguiente (figura 1). Se utilizó como herramienta de análisis para el análisis cualitativo el CAQDAS Nvivo12PLUS.

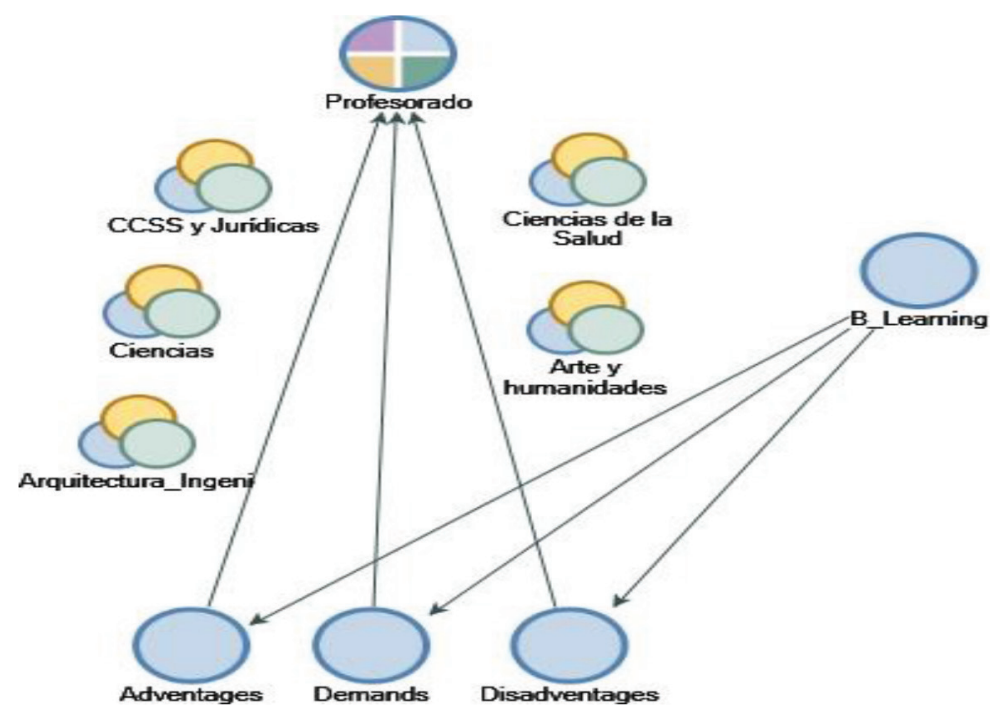

Figura 1 - Mapa conceptual de las opiniones del profesorado sobre la enseñanza B_learning

En la segunda fase del análisis se realizó la codificación garantizando la calidad del estudio mediante la valoración inter e intrajueces -credibilidad o validez interna(Sánchez Gómez y Martin García, 2017). Para probar la dependencia (fiabilidad) del sistema de categorías se elaboró un libro de códigos que facilitó la asignación textual a la categoría correspondiente por parate de los expertos. La objetividad (Confirmabilidad) se demuestra en los de fragmentos literales del discurso de los docentes. A partir de las definiciones conceptuales de los indicadores que forman parte del modelo TAM de la enseñanza b-learning tomadas de la literatura de referencia anteriormente citada, se identificaron las categorías en los textos (proceso de codificación). En la última fase del estudio se muestra el resultado de los análisis de contenido.

Además, como refuerzo a este análisis, se aplicó la técnica DAFO (Debilidades, Amenazas Fortalezas y Oportunidades) con la que se pretende integrar las diferentes decisiones llevadas a cabo de acuerdo con las premisas de la confiabilidad, para garantizar la objetividad y confirmabilidad de los resultados. Mediante la técnica DAFO se completa el diseño mixto, DEPLIX, ya que posibilita la integración de los resultados de la primera fase cuantitativa (utilidad percibida, facilidad percibida de uso, actitudes hacia la enseñanza b-learning, e intención de uso b-learning) con la segunda fase cualitativa (ventajas, inconvenientes y demandas del uso de B-learning en las enseñanzas universitarias). De 
esta forma, se realizan las conclusiones abordando el tema de investigación de forma completa e integral.

\section{Resultados}

\section{Diferencia entre grupos por ámbito disciplinar}

Los principales resultados obtenidos en el modelo empírico TAM muestran que la Intención de utilizar un sistema de formación basado en metodología mixta (BL) está determinada principalmente por las dos variables principales del modelo TAM, LAS expectativas de resultados o la Utilidad Percibida (UP) al implementar un sistema BL ( $\beta$ $=.519)$ y por la percepción del esfuerzo relativo o facilidad de uso que supondría utilizar este tipo de sistema $(\beta=.391)$. El contraste de la variable "ámbito disciplinar" con las variables del modelo, consideradas aquí de manera independiente, se realizó mediante Anova para valorar la existencia de diferencias entre los grupos. Así, en el caso de la intención de uso del sistema (IC_BL) se aprecia que existen diferencias significativas $(\mathrm{F}=4,42, \mathrm{p}=.002)$, mostrando los profesores de Ciencias una menor intención de uso de BL que los de Arte y Humanidades ( $\mathrm{p}=.003$ ), no apareciendo otras diferencias con el resto de ramas de conocimiento. Respecto a la variable Actitud hacia BL los profesores de Ciencias presentan puntuaciones medias significativamente más bajas $(\mathrm{F}=5,00$, $\mathrm{p}=.001)$ que los de Ciencias de la Salud ( $\mathrm{p}=.000)$, que los de Ciencias Sociales y Jurídicas ( $\mathrm{p}=.035)$ y que los de Arquitectura/Ingeniería $(\mathrm{p}=.026)$

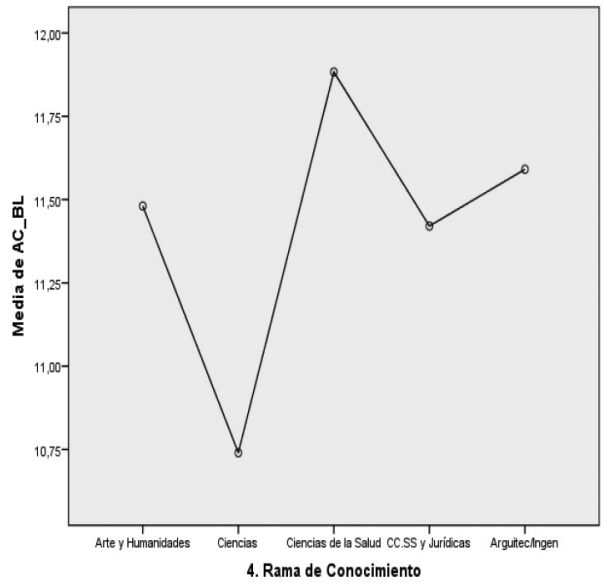

Figura 2 - Diferencias de medias en AC_BL por Ámbito disciplinar

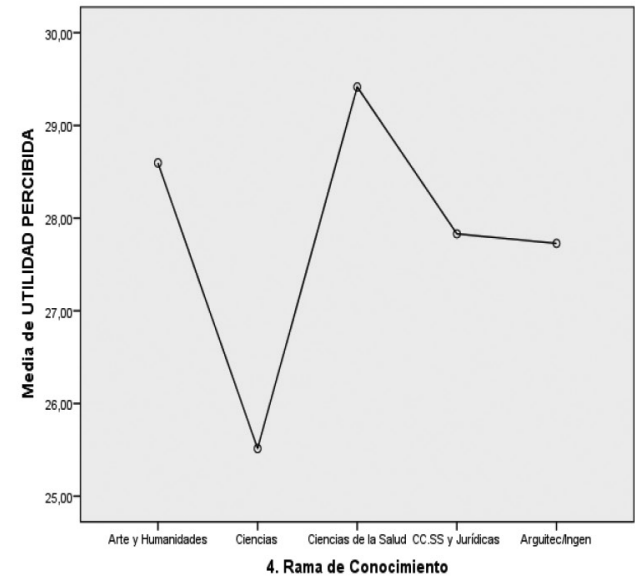

Figura 3 - Diferencias de medias en Utilidad Percibida por Ámbito disciplinar

En el caso de la variable Facilidad de uso percibido (PEOU) únicamente aparecen diferencias significativas $(\mathrm{F}=3,69, \mathrm{p}=$.005) entre los profesores de Arquitectura e Ingeniería que perciben menor dificultad en el uso de sistemas BL que los de Ciencias de la Salud ( $\mathrm{p}=.038)$. Por su parte, los profesores de Arte y Humanidades manifiestan 
percibir menores condiciones facilitadoras (CF), en cuanto a la existencia de recursos de apoyo o infraestructuras técnicas para el desarrollo de metodología BL en sus centros que los profesores de Ciencias ( $\mathrm{p}=.016)$ y que los de Ciencias Sociales y Jurídicas ( $\mathrm{p}=.003$ ).

Por último, respecto a la variable "Utilidad Percibida" (UP) se aprecian diferencias significativas $(\mathrm{F}=7,06, \mathrm{p}=.000)$ entre los profesores de Ciencias con los de Arte y Humanidades $(\mathrm{p}=.014)$, con los de Ciencias de la Salud $(\mathrm{p}=.000)$ y con los de Ciencias Sociales y Jurídicas $(\mathrm{p}=.049)$ valorando los de ciencias una menor utilidad de la metodología BL que los otros tres grupos

\section{Análisis cualitativo:}

Se presentan los resultados de los análisis de datos textuales siguiendo los siguientes indicadores: análisis del léxico (expresiones más frecuentes/significado/frecuencia ocurrencia) y análisis de contenido cualitativo Las dimensiones del análisis vienen definidas por ventajas, desventajas y demandas del uso metodología de enseñanza B_ learning. En los árboles de palabras en los que el programa Nvivo representa gráficamente las expresiones más relevantes se aprecian los siguientes comentarios.

Los comentarios ponen de manifiesto las ventajas y desventajas en relación con los estudiantes y tareas docentes, mientras que en las demandas centran su atención en las deficientes infraestructuras universitarias para poner en marcha estas metodologías. En resumen:

- $\quad$ Efectos de estas metodologías en el alumnado:

- $\quad$ Asignaturas más asequibles

- Favorecen la adaptación al ritmo de trabajo del estudiante

- Potencian la interacción profesor -alumno

- Incrementa la autonomía y responsabilidad

- Benefician el trabajo a distancia

- Dificultad para contabilizar sus horas de trabajo

- Conflicto con las técnicas convencionales de evaluación de los estudiantes

- Dificultad para identificar al estudiante (suplantación de identidad

- Riesgo a que pierdan el interés y motivación

- $\quad$ Efectos en los docentes:

- Obliga a una estructuración más compleja de las materias en cuanto a la presentación de forma práctica y coherente de los contenidos

- Favorece el trabajo cooperativo, significativo y colaborativo

- Excesivo número de horas para preparar las asignaturas en esta modalidad

- Falta de incentivos

- Necesidad de apoyo y formación

- Carencia de infraestructuras adecuadas y elevado número de estudiantes por profesor lo que en muchas ocasiones hace a este tipo de enseñanza inviable

Tras el análisis de contenido los docentes universitarios participantes en el estudio encuentran más inconvenientes que ventajas en uso de la metodología b-learnig en la enseñanza superior: 
El número de comentarios constatan en general más dificultades que ventajas en la utilización de la enseñanza B_L. Los docentes de las titulaciones de Ciencias, seguidos de las Ingenierías y Arquitectura; los que menos inconvenientes relatan son los de Arte y Humanidades. Por su parte, Ciencias de la Salud y CCSS y Jurídicas son los que comentan más factores positivos y los de CCSS y Jurídicas los que exponen más demandas. Además, menos en CCSS, Jurícicas y de la Salud, los demás docentes encuentran más dificultades que ventajas a la hora de implementar el tipo de enseñanza B_Learnig. Los factores positivos señalados por los docentes de Ciencias Sociales, Jurídicas y de la Salud son el acercamiento a la realidad social, facilidad en casos prácticos y gestión de la documentación, fortalecimiento de competencias digitales, autonomía personal y trabajo cooperativo, mayor interacción profesor -alumno y aprendizaje significativo. Además, potencia la comunicación y mejora los procesos de evaluación.

Las desventajas que subrayan los docentes de las titulaciones de Ciencias se refieren sobre todo en temas relacionados con la ratio tan elevada de estudiantes por grupo, más tiempo y esfuerzo personal, importancia del contacto presencial frente al virtual, pésima coordinación entre docentes, suplantación de personalidad entre estudiantes, dificultad para contabilizar las horas en las actividades que desarrolla el estudiante de forma no presencial, dificultad de integral estas actividades en el proceso de evaluación y la escasa motivación y el alto porcentaje de abandono de los estudiantes.

Por lo que se refiere a las demandas, los profesores de ingeniería y arquitectura reclaman política institucional, correctamente diseñada y financiada, que esté valorada en su justa medida, apoyo en infraestructuras y formación. Los de Ciencias Sociales y Jurídicas reclaman, además, normativa específica e iniciativas de formación docente desde las facultades (Martín et al., 2019)

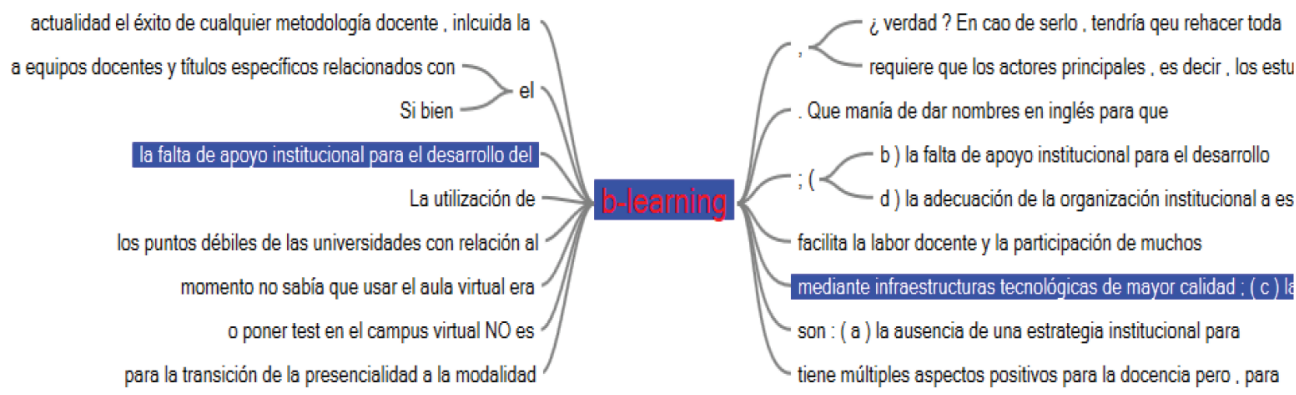

Figura 4 - Comentarios de los docentes 


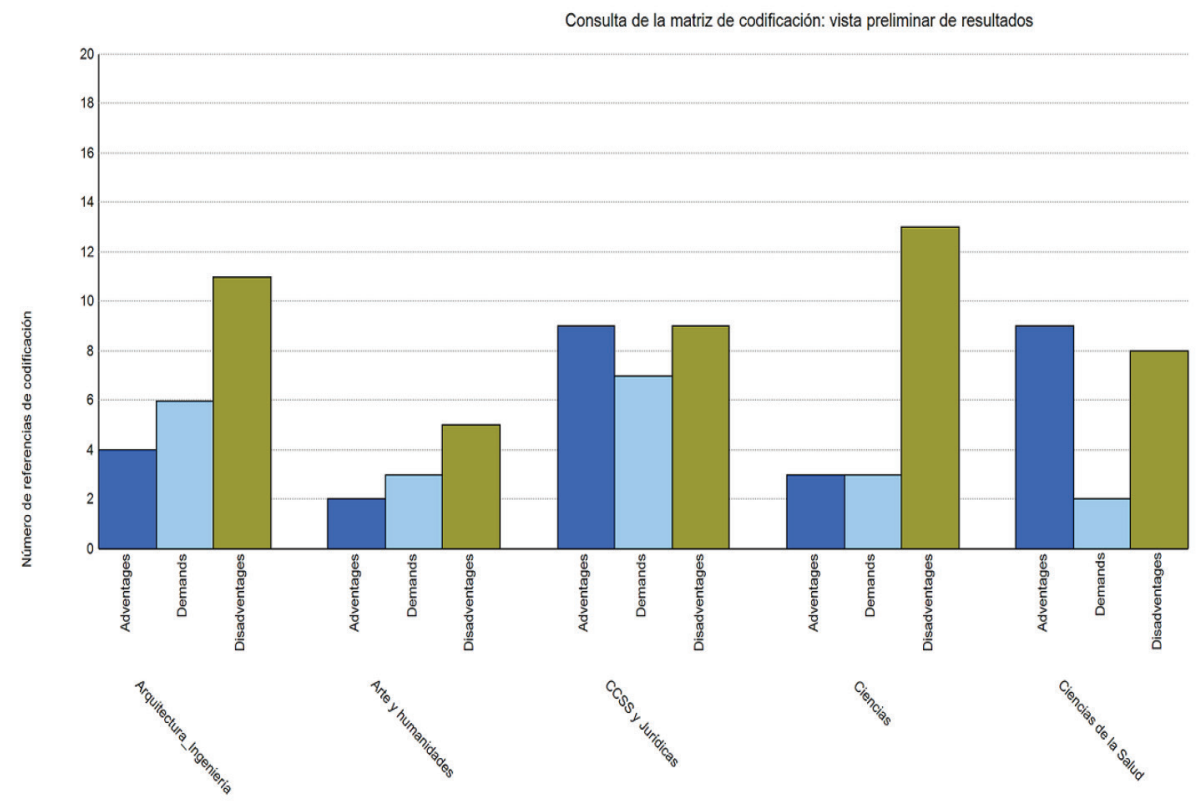

Figura 5 - Análisis de contenido (\% Referencias de codificación por ámbito disciplinar_ dimensiones)

\section{Análisis DAFO}

Para integrar los resultados de los análisis CUANT-CUAL se ha realizado un análisis DAFO en el que se ponen de manifiesto las debilidades, amenazas, fortalezas y oportunidades de la enseñanza B-learning, a nivel interno y externo de las instituciones universitarias en el que se desarrolla el estudio para potenciar su eficacia y eficiencia. En apartados anteriores se ha descrito la situación actual en opinión de los docentes, y así identificar las acciones o cambios que han de llevarse a cabo. Las fases en las que hemos realizado la DAFO son

1. análisis interno de la institución universitaria a juicio de los profesores y su percepción sobre b-learning. Debilidades: para corregirlas o minimizarlas y fortalezas: para impulsarlas y mantenerlas.

2. análisis externo: amenazas (afrontarlas, reducirlas o eliminarlas) yoportunidades (explotarlas). Son indicadores que no dependen directamente del profesorado pero que le afectan. Estos puntos son muy importantes para conocer la realidad, tomar decisiones, aprovechar nuevas oportunidades y diseñar estrategias a seguir. 


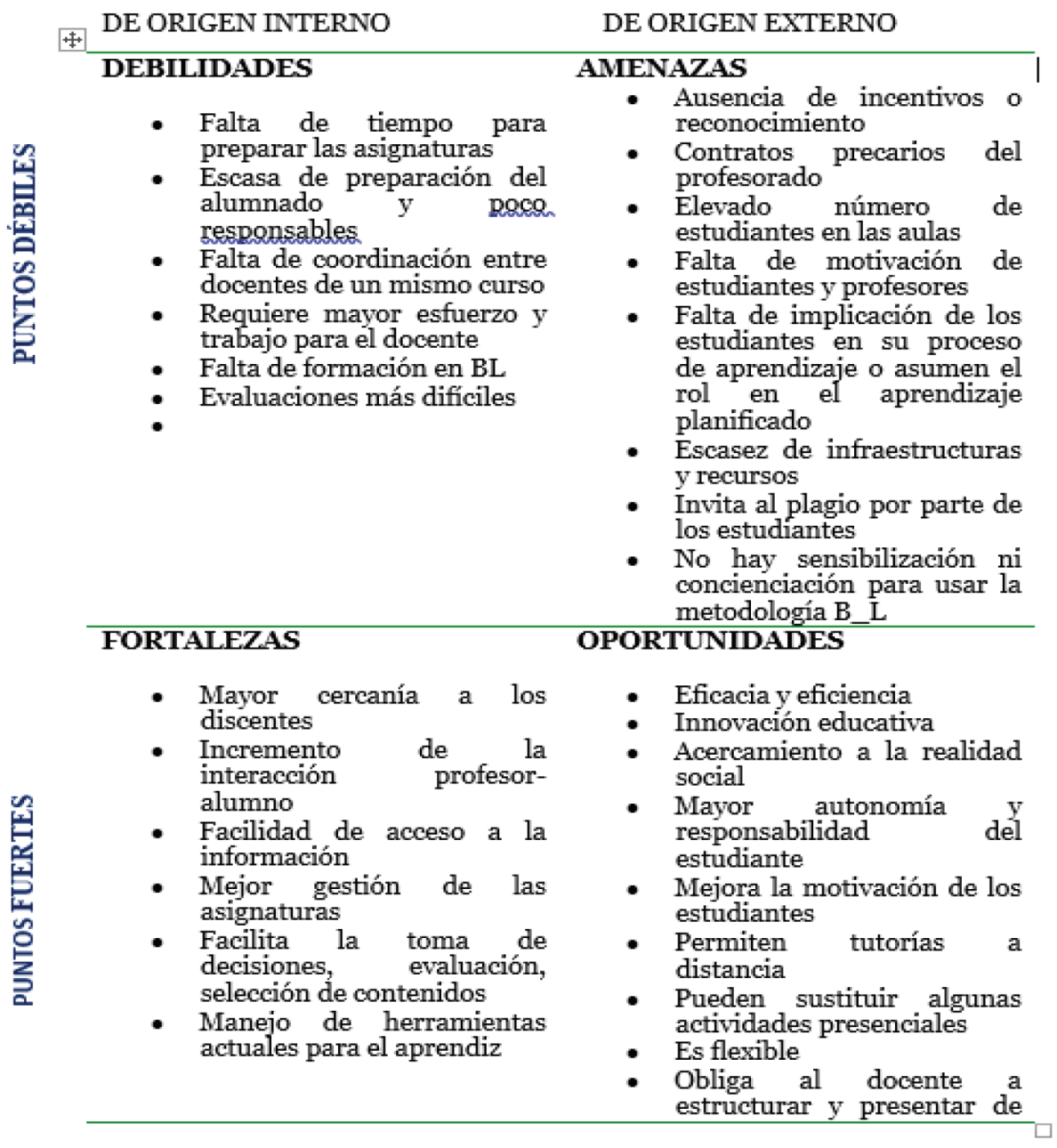

Tabla 1 - Resultados del análisis DAFO sobre percepciones profesores sobre BL

\section{Conclusiones}

El efecto conjunto de las variables principales del modelo TAM planteado tanto sobre la intención de utilizar la modalidad de enseñanza combinada (BL) como del Uso efectivo del mismo, prueba la idoneidad de este enfoque también para el estudio de conductas o sistemas complejos. Los resultados de nuestro trabajo apoyan la idea que el valor predictivo del modelo TAM está fundamentalmente en las variables principales (AT, PU, PEOU), resultado que está en línea con lo obtenido por meta-análisis recientes como el realizado por Scherer, Siddiq \& Teo, 2015) a partir del estudio de varias decenas de trabajos con TAM, poniendo de manifiesto que la mayor parte de extensiones al modelo apenas aportan una mejora significativa a su poder teórico respecto a las variables originales. 
De este modo, y a partir de los análisis efectuados en nuestro estudio, el mayor peso explicativo en la intención de uso de un sistema BL recae en la Actitud hacia el uso de este tipo de entornos $\left(70,6 \%, \mathrm{R}^{2}=, 706\right)$. Este resultado es de interés para muchas universidades e instituciones de educación superior, ocupadas en promover planes estratégicos para implementar y difundir BL. En este sentido resulta fundamental destacar la importancia que tiene que los profesores valoren positivamente la necesidad de integrar las modalidades formativas presencial y virtual, acentuando las ventajas en el uso de este sistema. Para ello, es necesario identificar una serie de creencias salientes sobre expectativas de resultados positivos derivados del uso de entornos BL (por ejemplo, en nuestro caso destacan: "La metodología BL agiliza la organización y presentación de la información y los contenidos de aprendizaje; "Hace más interesantes y amenas las asignaturas", "Contribuye a mejorar o hace más fácil el aprendizaje de los alumnos”, etc).

Asimismo, la segunda variable que modula la intención de uso de un entorno BL es la percepción sobre su facilidad o dificultad de uso, de modo que se asume que es más probable que un individuo la abandone cuando percibe que la innovación es más compleja que cuando la considera más fácil de usar (Aldunate \& Nussbaum, 2013). Este resultado es lógico y ha sido puesto de manifiesto en numerosos estudios empíricos. En el caso concreto de los entornos BL, y de manera general, existe una creencia generalizada sobre la complejidad añadida que conlleva utilizar una modalidad que, en muchas ocasiones, puede implicar duplicar procesos instruccionales. Hemos comprobado en nuestro trabajo que las expectativas que hacen percibir con mayor facilidad el uso de los entornos BL están relacionadas con la percepción de un adecuado apoyo institucional, material y técnico (condiciones facilitadoras), así como por el efecto positivo de la influencia de colegas y personas significativas en el entorno laboral.

Además de ello, la formación previa específica sobre el manejo de este tipo de entornos, tanto en aspectos tecnológicos como instruccionales es un factor también de importancia para incrementar su intención potencial de uso, como se pone de manifiesto en la literatura especializada. En particular, se aprecia la importancia de la experiencia previa en el uso de recursos digitales sobre la mejora de la percepción de utilidad de este tipo de sistemas, resultado que apoya algunos estudios en los que se ha observado que el éxito de e_learning o b-Learning tienen una clara relación con la familiaridad y manejo de plataformas y recursos tecnológicos aplicados al aula (Jorne, González-Such \& GarcíaBellido, 2012; Picciano \& Seaman, 2007).

En cuanto a los resultados encontrados respecto a diferencias en función del ámbito disciplinar (Rama de Conocimiento) parecen sugerir la necesidad de ampliar y realizar nuevos estudios que permitan valorar la consistencia o no de los mismos.

Finalmente, a nivel metodológico este estudio abre la puerta al uso de múltiples métodos en la investigación, puesto que obliga a una amplia gama de posibles técnicas de recogida de información y de análisis. Ante problemas complejos, como puede ser el estudio de la formación basada en diseños blended learning, el investigador precisa seleccionar opciones metodológicas en un amplio y diverso campo temático, lo que implica una pluralidad metodológica que diversifica los modos de aproximación, descubrimiento y justificación en atención a la faceta o dimensión del objeto educativo que se estudia. En nuestro estudio hemos prestado atención a un aspecto muy específico relacionado con 
la percepción de la metodología BL de profesores universitarios de distintos ámbitos disciplinares, pero el estudio demuestra que la investigación con métodos mixtos puede desempeñar un papel clave para dar una visión holística y exhaustiva de la realidad estudiada.

\section{Agradecimientos}

Este estudio ha sido realizado con el apoyo del Ministerio de Economía y Competitividad de España y el Fondo Europeo de Desarrollo Regional (FEDER). Plan I+D+i, Proyecto Ref. EDU2015-6721

\section{Referencias}

Al-Azawei, A., Parslow, P., \& Lundqvist, K. (2017). Investigating the effect of learning styles in a blended e-learning system: An extension of the technology acceptance model (TAM). Australasian Journal of Educational Technology, 33(2).

Al-Busaidi, K. A. (2013). An empirical investigation linking learners' adoption of blended learning to their intention of full e-learning. Behaviour \& Information Technology, 32(11), 1168-1176. DOI: https ://doi.org/10.1080/01449 29X.2013.774047

Aldunate, R., \& Nussbaum, M. (2013). Teacher adoption of technology. Computers in Human Behavior, 29(3), 519-524. DOI: https://doi.org/10.1016/j.chb.2012.10.017

Aliyu, O., Arasanmi, C. C., \& Ekundayo, S. (2019). Do demographic characteristics moderate the acceptance and use of the Moodle learning system among business students?. International Journal of Education and Development using ICT, 15(1).

Barrera-Barrera, R., Rey-Moreno, M., \& Medina-Molina, C. (2019). Fatores explicativos da preferência e uso da administração eletrônica na Espanha. Revista de Administração Pública, 53(2), 349-374. DOI: http://dx.doi.org/10.1590/o034761220170391

Bliuc, A. M., Casey, G., Bachfischer, A., Goodyear, P., \& Ellis, R. A. (2012). Blended learning in vocational education: teachers' conceptions of blended learning and their approaches to teaching and design. The Australian Educational Researcher, 39(2), 237-257.

Boelens, R., Voet, M., \& De Wewer, B. (2018). The design of blended learning in response to student diversity in higher education: Instructors' views and use of differentiated instruction in blended learning. Computers \& Education, 120, 197-212. DOI: https://doi.org/10.1016/j.compedu.2018.02.009

Cavanaugh, C., Hargis, J., \& Mayberry, J. (2016). Participation in the Virtual Environment of Blended College Courses: An Activity Study of Student Performance. International Review of Research in Open and Distributed Learning, 17(3), 263-275. doi: http:// dx.doi.org/10.19173/irrodl.v17i3.1811

Chen, J. L. (2011). The effects of education compatibility and technological expectancy on e-learning acceptance. Computers \& Education, 57, 1501-1511. 
Davis, F. D., Bagozzi, R. P., \& Warshaw, P. R. (1989). User acceptance of computer technology: a comparison of two theoretical models. Management science, 35(8), 982-1003.

Dziuban, C., Graham, C. R., Moskal, P. D., Norberg, A., \& Sicilia, N. (2018). Blended learning: The new normal and emerging technologies. International Journal of Educational Technology in Higher Education, 15, 1-16. doi: http://dx.doi. org/10.1186M1239-017-0087-5

Ginns, P., \& Ellis, R.A. (2009). Evaluating the quality of e-learning at the degree level in the student experience of blended learning. British Journal of Educational Technology, 40(4), 652-663.

Graham, C. R., Woodfield, W., \& Harrison, J. B. (2013). A framework for institutional adoption and implementation of blended learning in higher education. The internet and higher education, 18, 4-14.

Ilic, D., Hart, W., Fiddes, P., Misso, M., \& Villanueva, E. (2013). Adopting a blended learning approach to teaching evidence based medicine: a mixed methods study. BMC medical education, 13(1), 169.

Ilic, D., Nordin, R. B., Glasziou, P., Tilson, J. K., \& Villanueva, E. (2013). Implementation of a blended learning approach to teaching evidence based practice: a protocol for a mixed methods study. BMC medical education, 13(1), 170.

Imtiaz, M. A., \& Maarop, N. (2014). A review of technology acceptance studies in the field of education. Jurnal Teknologi, 69(2).

Jornet Meliá, J., González-Such, J., \& García-Bellido, A. (2012). La Investigación Evaluativa y las Tecnologías de la Información y la Comunicación (TIC). Revista Española de Pedagogía, 251, 93-110.

Kim, H. W., Kankanhalli, A., \& Lee, H. L. (2016). Investigating decision factors in mobile application purchase: A mixed-methods approach. Information \& Management, 53(6), 727-739.

Konak, A., Kulturel-Konak, S., Nasereddin, M., \& Bartolacci, M. R. (2017). Impact of Collaborative Work on Technology Acceptance: A Case Study from Virtual Computing. Journal of Information Technology Education, 16(1), 15-19. Doi: https://doi.org/10.28945/3622

Lai, M., Lam, K. M., \& Lim, C. P. (2016). Design principles for the blend in blended learning: a collective case study. Teaching in Higher Education, 21(6), 716-729. Doi: doi.org/10.1080/13562517.2016.1183611

Lee, B. C., Yoon, J. O., \& Lee, I. (2009). Learners' acceptance of e-learning in South Korea: Theories and results. Computers \& Education, 53(4), 1320-1329.

Lee, Y., Kozar, K. A., \& Larsen, K. R. (2003). The technology acceptance model: Past, present, and future. Communications of the Association for information systems, 12(1), 50. DOI: 10.17705/1CAIS.01250 
Lim, D. H., \& Morris, M. L. (2009). Learner and Instructional Factors Influencing Learning Outcomes within a Blended Learning Environment. Educational Technology \& Society, 12 (4), 282-293.

Marangunić, N., \& Granić, A. (2015). Technology acceptance model: a literature review from 1986 to 2013. Universal Access in the Information Society, 14(1), 81-95.

Martín-García, A.V., \& Sánchez-Gómez, M.C. (2014). Modelo predictivo de la intención de adopción de Blended learning en profesores universitarios. Universitas Psychologica, 13(2), 601-614.

Martín-García, A.V., Martínez Abad, F., \& Reyes González, D. (2019). TAM and stages of adoption of blended learning in higher education by application of data mining techniques. British Journal of Educational Technology, 50, 2484-2500. Doi: https://doi.org/10.1111/bjet.12831

Martín-García, A.V., Sánchez-Gómez, M. C., \& Costa, P. (2019) Percepción de Blended Learning en profesores universitarios de distintos ámbitos disciplinares. Revista Lusófona de Educação, 44, 117-133. Doi: 10.24140/issn.1645-7250.rle44.o

Ngai, E. T., Poon, J. L., \& Chan, Y. C. (2007). Empirical examination of the adoption of WebCT using TAM. Computers \& Education, 48(2), 250-267. Doi: 10.1016/j. compedu.2004.11.007

Picciano, A. G., \& Seaman, J. (2007). K-12 online learning: a survey of US school district administrators. Boston: Sloan Consortium. Retrieved from: https://bit.ly/2TPb103

Poon, J. (2013). Blended learning: An institutional approach for enhancing students' learning experiences. Journal of online learning and teaching, 9(2), 271-288.

Pynoo, B., Tondeur, J., Van Braak, J., Duyck, W., Sijnave, B., \& Duyck, P. (2012). Teachers' Acceptance and Use of an Educational Portal. Computers \& Education, 58(4), 1308-1317. Doi: https://doi.org/10.1016/j.compedu.2011.12.026

Pynoo, P., Devolder, J., Tondeur, J., Van Braak, W., \& Duyck, P. (2011). Predicting secondary school teachers' acceptance and use of a digital learning environment: A cross-sectional study. Computers in Human Behavior, 27 (1), 568-575. Doi: 10.1016/j.chb.2010.10.005

Scherer, R., Siddiq, F., \& Teo, T. (2015). Becoming more specific: Measuring and modeling teachers' perceived usefulness of ICT in the context of teaching and learning. Computers \& Education, 88, 202-214. Doi: https://doi.org/10.1016/j. compedu.2015.05.005

Scherer, R., Siddiq, F., \& Tondeur, J. (2019). The technology acceptance model (TAM): A meta-analytic structural equation modeling approach to explaining teachers' adoption of digital technology in education. Computers \& Education, 128, 13-35. Doi: https ://doi.org/10.1016/j.compe du.2018.09.009

Selim, H. M. (2007). Critical Success Factors for E-Learning Acceptance: Confirmatory Factor Models. Computers \& Education, 49(2), 396-413. 
Šumak, B., \& Šorgo, A. (2016). The acceptance and use of interactive whiteboards among teachers: Differences in UTAUT determinants between pre- and post-adopters. Computers in Human Behavior, 64, 602-620. Doi: https://doi.org/10.1016/j. chb.2016.07.037

Teo, T. (2011). Factors Influencing Teachers' Intention to Use Technology: Model Development and Test. Computers \& Education, 57(4), 2432-2440.

Teo, T., Fan, X., \& Du, J. (2015). Technology acceptance among pre-service teachers: Does gender matter?. Australasian Journal of Educational Technology, 31, 235251. DOI: https://doi.org/10.14742/ajet.1672 https://bit.ly/2Cm8vi7

Venkatesh, V., Morris, M. G., Davis, G. B., \& Davis, F. D. (2003). User acceptance of information technology: Toward a unified view. MIS Quarterly, 27(3), 425-478. Doi: https://bit.ly/2kFEjEE

Venkatesh, V., \& Bala, H. (2008). Technology acceptance model 3 and a research agenda on interventions. Decision Sciences, 39(2), 273-315.

Venkatesh, V., \& Davis, F. D. (200o). A theoretical extension of the technology acceptance model: Four longitudinal field studies. Management science, 46(2), 186-204.

Venkatesh, V., Brown, S. A., Maruping, L. M., \& Bala, H. (2008). Predicting different conceptualizations of system use: the competing roles of behavioral intention, facilitating conditions, and behavioral expectation. MIS quarterly, 32(3), 483-5.

Wu, P. F. (2011). A mixed methods approach to technology acceptance research. Journal of the AIS, 13(3), 172-187. 